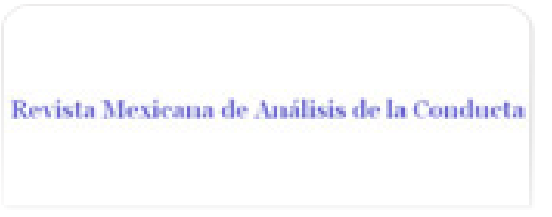

Revista Mexicana de Análisis de la Conducta ISSN: 0185-4534

editora@rmac-mx.org

Sociedad Mexicana de Análisis de la Conducta México

Vite Sierra, Ariel; Pérez Granados, Ignacio; Ruiz Cabello, Mireya El impacto de la sensibilidad materna y el entrenamiento a padres en niños con problemas de conducta

Revista Mexicana de Análisis de la Conducta, vol. 34, núm. 2, diciembre, 2008, pp. 165-178 Sociedad Mexicana de Análisis de la Conducta

Guadalajara, México

Disponible en: http://www.redalyc.org/articulo.oa?id=59311115003

Cómo citar el artículo

- Número completo

- Más información del artículo

Página de la revista en redalyc.org

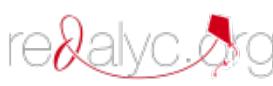

Sistema de Información Científica Red de Revistas Científicas de América Latina, el Caribe, España y Portugal Proyecto académico sin fines de lucro, desarrollado bajo la iniciativa de acceso abierto 


\title{
EL IMPACTO DE LA SENSIBILIDAD MATERNA Y EL ENTRENAMIENTO A PADRES EN NIÑOS CON PROBLEMAS DE CONDUCTA
}

\author{
THE IMPACT OF MATERNAL RESPONSIVENESS AND PARENT \\ TRAINING WITH CONDUCT-DISORDERED CHILDREN
}

\author{
ARIEL VITE SIERRA, IGNACIO PÉREZ GRANADOS \\ Y MIREYA RUIZ CABELLO' \\ FACULTAD DE PSICOLOGÍA, UNAM
}

\begin{abstract}
RESUMEN
El interés del presente trabajo fue evaluar el efecto que tiene el reflejo y la aprobación social en promover la sensibilidad materna en madres de niños con problemas de conducta. A través de un diseño experimental de un solo sujeto, se instrumentó un programa de intervención conductual que empleó instrucciones, modelamiento y retroalimentación visual. Los resultados muestran un incremento significativo en el índice de sensibilidad materna y una reducción del comportamiento infantil aversivo. Estos hallazgos se discuten en términos del impacto de la sensibilidad materna en el entrenamiento a padres de niños con problemas de conducta.

Palabras Clave: Problemas de conducta, sensibilidad materna, díadas madre-hijo, desobediencia, entrenamiento a padres.
\end{abstract}

\begin{abstract}
We evaluated the effect of the mirroring and the social approval to promote the maternal responsiveness in children's mothers with behavior problems. A
\end{abstract}

1. Los autores agradecen a los revisores anónimos sus atinados comentarios.

Para correspondencia dirigirse a Ariel Vite correo electrónico: avite@servidor.unam.mx Recibido: 31 Marzo, 2008. Revisado: 13 de Agosto, 2008. Aceptado: Diciembre 28, 2008 
program of behavioral intervention was instrumented through a single case experimental design using instructions, modeling and visual feedback. The results showed a significant increment in the index of maternal responsiveness and a reduction of the aversive behavior of the child. These findings are discussed in terms of the impact of the maternal sensibility in the training to parents in children with behavior problems.

Key words: Problem behavior, mothers' responsiveness, mother- child dyads, child compliance, training parents.

Los problemas de comportamiento infantil pueden representar un serio desafío a las familias, escuelas y sociedad, ya que una vez establecidos, tienden a persistir y la falta de tratamiento los multiplica, intensifica y diversifica con el tiempo (Campbell \& Ewing, 1990; Kazdin, 2000; Webster-Stratton, 2000). Este hecho puede situar al niño en riesgo de fracaso académico, aislamiento social y rechazo por parte de compañeros. Asimismo, incrementa la probabilidad de deserción escolar, alcoholismo y abuso de drogas, (Dodge, 1993; lalongo, Poduska, Werthamer \& Kellam, 2001; Kaiser, Cai, Hancock \& Foster, 2002; Kazdin, 1993).

Por otra parte, los padres de niños con problemas de conducta, tienden a etiquetar la conducta infantil como desviada y es más probable que respondan a la conducta positiva y neutral de sus hijos de manera negativa (Dix, 1991; Kochanska, 1992; Lytton, 1990). Se ha demostrado, además, que estos padres inician menos contacto social cuando sus niños están involucrados en actividades solitarias e invierten menos tiempo en juegos cooperativos y actividades conjuntas, que los padres de niños "normales" (Wahler, Castellani, Smith \& Keathley, 1996). Además, la probabilidad de que los padres respondan a la conducta infantil inadecuada con castigos o ignorándola, es alta y asimismo, no intentan emplear otro tipo de estrategias disciplinarias (Snyder, Edwards, McGraw, Kilgore \& Holton, 1994). Por otra parte, son inconsistentes en hacer cumplir las normas e instrucciones y es más probable que respondan a las protestas del niño y a su conducta inadecuada de la misma forma o cediendo ante ésta (Serna, Nielsen, Lambros \& Forness, 2000; Snyder \& Patterson, 1995).

Los estudios antes mencionados, han formado una extensa base empírica que sostiene la hipótesis de que cuando los padres son entrenados para implementar estrategias de cambio conductual, mejoran sus interacciones y por lo tanto, el ajuste social y emocional del niño.

Al respecto, los padres son capacitados para mantenerse firmes al enfrentar los intentos coercitivos del niño con la finalidad de no reforzarlos (Gelfand \& Hartmann, 1984; Kaiser \& Hester, 1997) y se les enseñan técnicas de manejo de contingencias no corporales, como el tiempo-fuera, para reducir la 
conducta aversiva infantil. Además de alterar las contingencias para reducir las conductas no deseadas, la aproximación conductual emplea técnicas de manejo de contingencias para incrementar la frecuencia de las conductas apropiadas. Por ejemplo, además de enseñar a los padres a reducir el reforzamiento de conducta negativa, también se les enseña a incrementar el reforzamiento social para las conductas positivas. Este procedimiento ha tomado la forma de "pescar al niño portándose bien" con el fin de reforzar esas conductas (Lavigueur, Tremblay \& Saucier, 1995; Shelton, Frick \& Wooten, 1996).

En contraste, los estudios de los intercambios madre-hijo presuponen que la obediencia del niño depende de la reciprocidad diádica, recurriendo a la variable molar, sensibilidad, para su explicación. La sensibilidad es un constructo que describe el comportamiento de los individuos quienes se involucran en interacciones diádicas. Cuando se aplica al dominio paternal, se define como las reacciones oportunas y apropiadas a las conductas y verbalizaciones del niño (Isabella, Belsky \& von Eye, 1989; Parpal \& Maccoby, 1985, Strand, 2000a, 2000b; Wahler \& Meginnis, 1997; Westerman, 1990). Los niños que tienen madres sensibles reaccionan de manera positiva y cooperativa hacia ellas (Kochanska, 2002; Kochanska \& Murray, 2000).

Mientras que los niños de madres que no son sensibles están propensos a mostrar conducta oposicional y afecto negativo (Johnston, Murray, Hinshaw, Pelhman \& Hoza, 2002; Westerman, 1990). Es decir, una madre sensible a las señales de su hijo genera interacciones sincrónicas y presupone ser la piedra de toque de la conducta cooperativa del niño.

De acuerdo a Maccoby (1984), los padres sensibles generan en sus hijos la disposición a la reciprocidad, significando que el niño trabaja para mantener esta forma de apoyo paternal. Esta suposición fue confirmada por los trabajos de Kochanska sobre la "orientación mutuamente sensible" madrehijo, orquestada por la madre quién tiende a propiciar reacciones apropiadas y oportunas en relación al comportamiento infantil (Kochanska, 1997, 2002; Kochanska \& Murray, 2000). La hipótesis de direccionalidad, de este proceso, ha sido confirmada en dos estudios adicionales en los cuales se establece que la sensibilidad de las madres promueve la cooperación del niño (Kochanska, 1997; Kochanska \& Murray, 2000).

Al respecto, Wahler y Meginins (1997), señalan que desde la perspectiva de la teoría del reforzamiento, la sensibilidad materna puede propiciarse a través de enseñar a las madres a proporcionar respuestas específicas que contengan aprobaciones y/o información de manera selectiva posterior al comportamiento prosocial de sus hijos. La lógica de esta propuesta está basada en evidencia empírica que muestra que la aprobación (e.g. "Buen trabajo") o reflejo (información) (e.g. "Veo que terminaste tu trabajo") frecuentemente funcionan como reforzadores positivos. De este modo, al proporcionar 
una forma específica de retroalimentación social para las conductas prosociales infantiles la madre está en posibilidad de generar sincronía a través del arreglo de otras contingencias sociales apropiadas al amplio repertorio del comportamiento infantil.

A fin de evaluar esta propuesta llevaron a cabo dos estudios; en el primero, analizaron el efecto que tiene el empleo de la aprobación y reflejo de manera separada en un grupo de 36 díadas madre-hijo con problemas de comportamiento. Cada díada fue asignada a uno de los tres grupos (reflexión, aprobación o control). Las madres asignadas al grupo de reflejo, escucharon las descripciones verbales de la reflexión por parte del entrenador y después observaron un video tape en donde un modelo mostraba como realizar la táctica durante el juego. El entrenador subrayaba el afecto neutral del modelo y la descripción breve o parafraseo de la conducta verbal o no verbal (e.g. "Así que prefieres el carro rojo").

En el grupo de aprobación, el entrenador enfatizaba el uso de afecto positivo presente a través de declaraciones ambiguas (e.g. "Wow, esto es sensacional"), y estas madres observaban un video instruccional que modelaba el uso efectivo de alabanzas. En contraste con la reflexión, la alabanza comprendió solamente la aprobación en tono y contenido, evitando la información sobre la respuesta del niño. Es decir, la reflexión y las alabanzas fueron definidas de manera mutuamente excluyente. En la cual la información y el afecto fueron separados.

Los resultados obtenidos señalan que las madres de los grupos experimentales (reflejo y aprobación) proporcionaron en las sesiones de juego libre, comentarios de apoyo más frecuentes y sus hijos mostraron reciprocidad a través de altas probabilidades de obediencia. Considerando estos datos, parece que la reflexión y la alabanza sirven como reforzamiento positivo y que su contingencia posibilita en el niño fortalecer su probabilidad de obediencia (Wahler \& Meginins, 1997).

En el segundo estudio, Wahler y Bellamy (1997) entrenaron a dos madres de niños con problemas de conducta, a través de la enseñanza de dos procedimientos: obediencia y sensibilidad materna. La enseñanza de la obediencia se enfocó en el empleo por parte de las madres de instrucciones y consecuencias sociales dependiendo de las respuestas del niño. Las instrucciones fueron descritas como órdenes para que el niño iniciara o dejara de hacer alguna actividad, y una vez emitida, se le proporcionaba de manera contingente aprobación por obedecer y tiempo fuera por desobedecer.

El entrenamiento en sensibilidad materna consistió en enseñar a las madres, a través de la supervisión de las actividades de sus hijos, a igualar o ser consistente con el comportamiento de estos. Por ejemplo, un niño quien colorea varias frutas, se le podría ofrecer un comentario de reconocimiento al seleccionar el color correcto ("Ios limones son verdes"), es decir, se ofrece 
reconocimiento y promueven las conductas apropiadas que realiza el niño. Por otra parte, para la enseñanza de la obediencia se emplearon las instrucciones, con aprobaciones contingentes a su cumplimiento y el tiempo fuera se utilizó si el niño rehusaba cumplir la instrucción.

Los efectos del entrenamiento mostraron que la participación sincrónica de las madres en la conducta de juego y trabajo de sus hijos fue correlacionada con la reciprocidad infantil, hallazgos similares reportan el impacto positivo de la sensibilidad materna sobre las acciones prosociales de niños normales (Harrist, Pettit, Dodge \& Bates, 1994; Westerman, 1990).

Como se observa en los estudios descritos, las formas específicas de retroalimentación maternal, aprobación y reflejo, funcionan como reforzadores que posibilitan englobar el repertorio de la conducta infantil y satisfacer mutuamente las necesidades en la interacción madre-hijo. Por lo que la enseñanza de la reflexión y aprobación podría ser estructurada como un entrenamiento específico en sensibilidad, en virtud de que se espera que genere un patrón amplio de reciprocidad infantil.

Considerando los señalamientos anteriores, el objetivo fue evaluar el efecto que tiene la aprobación social y el reflejo en propiciar la sensibilidad materna en madres de niños con problemas de conducta.

\section{MÉTODO}

\section{Participantes}

Los participantes fueron nueve díadas madre-niño de clase media baja. Los niños presentaban problemas de conducta (desobediencia), y fueron detectados por las maestras del preescolar al que asistían. La muestra comprendió cinco niñas y cuatro niños, con una edad promedio de 5.2 años y una desviación estándar de 1.48 y la edad promedio de las madres fue de 31 años, con una desviación estándar de 3.18.

Los valores de riesgo clínico, lo cuales sugieren las fuentes de estrés y disfunción potencial del sistema padre-hijo, obtenidos con la aplicación del Índice de Estrés Paternal (Ayala, Pedroza, Morales, Chaparro \& Barragán, 2002), fueron en las subescalas de la dimensión del niño, Distractibilidad/ Hiperactividad $37.5(S D=5.150)$ y adaptabilidad $29.9(S D=2.86)$. Para la dimensión de los padres, en sentido de competencia se obtuvo $34(S D=4.2)$ y en la subescala situacional se obtuvo en a) restricción del rol $23.6(\mathrm{SD}=2.3)$ y b) aislamiento social $17.2(\mathrm{SD}=1.3)$. 
Escenario

Las sesiones se desarrollaron en un salón de $4 \times 4 \mathrm{~m}$ debidamente iluminado y exento de distractores externos, con mesas y sillas para niños de un jardín de niños ubicado en el sur de la Ciudad de México

Medición

Se utilizó el programa de cómputo SIRECC (Torres, Zarabozo \& López, 1991), que es un sistema de observación para el estudio de la interacción madre-niño en ambientes controlados. Sus principales características son: permite un registro continuo de las interacciones, posibilita la codificación secuencial de eventos, permite la obtención de medidas de frecuencia por sesión de cada código tanto de la madre como del hijo y sus tasas de respuesta por minuto.

Las observaciones fueron realizadas por observadores capacitados, quienes emplearon el Sistema Observacional de la Interacción Madre-Niño (SOI) (Vite \& Parra, 2002), el cual abarca un conjunto de categorías de comportamiento, mutuamente excluyentes y colectivamente exhaustivas, de la madre y del niño.

Las Conductas a evaluar fueron dos aspectos de la conducta materna y dos del comportamiento infantil. También se valoró el constructo de sensibilidad materna:

1. C conductas maternas:

Aversivas: comprendió las conductas de amenazar, desaprobar y regañar.

Reflejo: incluyó descripciones verbales de la madre hacia la conducta no verbal del niño o el parafraseo de su conducta verbal, empleando un tono de voz neutral y carente de un contenido de aprobación.

Aprobación: incluyó reconocimientos verbales positivos de las características del niño los cuales no eran referentes a la conducta que estaba realizando.

Así como dos aspectos del comportamiento infantil

2. Conductas infantiles:

Prosociales: abarco las conductas de obedecer y realizar la actividad.

Aversivas: comprendió las conductas de desobedecer y repelar. (Ver anexo 1 para la definición de las conductas) y el constructo de

\section{Sensibilidad Materna:}

Suma de intervalos de observación de las reacciones maternas apropiadas, contingentes a las conductas infantiles (prosociales y aversivas), dividido entre la suma de intervalos de respuestas maternas apropiadas e inapropiadas a las conductas infantiles (prosociales y aversivas). 
Para considerar el empleo apropiado de las aprobaciones y el reflejo, se requirió que éstas se presentaran de manera contingente a la ocurrencia de la conducta infantil (p.e. realizar la actividad, obedecer), pero no a la ocurrencia de desobedecer y repelar del niño, lo cual se basó en la regla de valencia común formulada y evaluada por Wahler y Dumas (1989) y Wahler, Williams y Cerezo (1990).

\section{Procedimiento}

Las fases de línea base, entrenamiento y seguimiento, constituyeron un diseño experimental intrasujeto $A B C$. En cada una de estas fases se programaron actividades relativas a la realización de la tarea escolar de los niños, con el propósito de propiciar que las madres se involucraran en dicha actividad. Se les indicó a las madres que auxiliaran a sus hijos en la realización de sus de sus tareas.

Línea base: En esta fase las nueve díadas fueron video grabadas en tres sesiones semanales de 20 minutos cada una. En esta condición se solicitó a las madres supervisar la realización de la tarea escolar de su hijo.

Entrenamiento: El entrenamiento se inició cuando el experimentador describió y ejemplificó el empleo de la aprobación social y del reflejo. Posteriormente, la madre y experimentador revisaron las video grabaciones de la línea base con la finalidad de identificar el momento en el cual deberían de emplearse, de manera contingente, dichas conductas. Cuando la madre indicaba de manera confiable cuándo emplear estas dos conductas (definida la confiabilidad como la concordancia de $90 \%$ con el experimentador), se le solicitó que llevara a la práctica lo aprendido al interactuar con su hijo en la realización de la tarea escolar. La sesión de la interacción de la madre con su hijo fue igualmente video grabada. Al final de la sesión el experimentador proporcionaba retroalimentación, y/o modelaba a la madre la manera de llevar a cabo la aprobación y el reflejo, dependiendo de su ejecución. Las sesiones subsecuentes se iniciaron, revisando la videograbación de la ejecución de la sesión anterior de cada madre y se seguía la dinámica descrita anteriormente. Esta fase tuvo una duración aproximada de 7 a 10 sesiones, con una promedio de 50 minutos por sesión.

Seguimiento: se realizó en tres sesiones, una cada semana, al finalizar el entrenamiento. Durante estas sesiones no se proporcionó instrucción o retroalimentación alguna a la madre. 


\section{RESULTADOS}

\section{Concordancia}

El acuerdo interobservador se obtuvo del $30 \%$ del total de las observaciones a través de las videograbaciones. Se empleó el coeficiente Kappa de Cohen (Fleiss, 1981). La concordancia entre observadores de las conductas de las madres en la Línea base fue de .78 a .85 y en el seguimiento de .80 a .84; la concordancia entre observadores para las conductas de los niños fue de .80 a .89 en la Línea base y de .79 a .88 en el seguimiento.

Para el análisis de los datos se llevó a cabo el siguiente proceso: los archivos en tiempo real fueron transformados en secuencias de comportamiento, de acuerdo con un muestreo temporal segundo a segundo. Posteriormente, los archivos de datos, de la madre y del niño se transformaron en uno solo, de forma que una secuencia de comportamiento fue la variable correspondiente a la madre y otra al hijo. Los datos resultantes se analizaron a través del paquete estadístico Statistica, a fin de obtener los tiempos dedicados por las madres y los niños a los diferentes comportamientos considerados para el estudio y determinar los cambios significativos entre la línea base y el seguimiento.

A fin de evaluar el efecto de las conductas maternas de aprobación, reflejo, y la sensibilidad materna, e infantiles prosociales y aversivas, se obtuvo el tiempo asignado por la madre y el hijo a cada categoría.

Los datos obtenidos en segundos de comportamiento, fueron transformados en minutos. Con los valores obtenidos se aplicó la prueba no paramétrica de pares de Wilcoxon, para cada una de las categorías de interés tanto de las madres como de los niños.

La Tabla 1 muestra los datos de las conductas de las madres en las fases de línea base y seguimiento, como se observa el promedio de la conducta aversiva en la línea base fue de 2.7 minutos y 1.01 en el seguimiento con una $p$ asociada .00. Por lo que respecta a la conducta de reflejo su promedio durante la línea base fue de 0.17 minutos en la línea base y 1.82 en el seguimiento, con una ganancia de 1.65 minutos con una probabilidad asociada de .00. Por último la aprobación materna se presento con un promedio de .05 durante la línea base y 1.85 durante el seguimiento, siendo la diferencia significativa de 00 .

Por lo que respecta al comportamiento infantil en la Tabla 2 se muestran los resultados obtenidos antes y después de la intervención, como se aprecia la conducta prosocial se presento en promedio 4.04 minutos en la línea base y 4.08 durante el seguimiento, no siendo la diferencia estadísticamente significativa. Por último el comportamiento aversivo tuvo un promedio en la línea base de 0.56 minutos y 0.04 en la fase de seguimiento, siendo la ganancia significativa al nivel de .00 . 


\begin{tabular}{lcccc}
\hline Conductas & Línea base & Seguimiento & $z$ & $p$ \\
\hline \multirow{2}{*}{ Aversivas } & 2.7 & 1.01 & 82.78 & .00 \\
\multirow{2}{*}{ Reflejo } & $(1.05)$ & $(.09)$ & & \\
& .17 & 1.82 & 71.14 & .00 \\
Aprobación & $(.05)$ & $(.17)$ & & \\
& .05 & 1.81 & 73.14 & .00 \\
\hline
\end{tabular}

Tabla 1. Media de tiempo (minutos) de las conductas maternas aversivas, aprobación y reflejo, en las fases de línea base y seguimiento. Entre paréntesis la desviación estándar.

\begin{tabular}{lcccc}
\hline Conductas & Línea Base & Seguimiento & $z$ & $p$ \\
\hline \multirow{2}{*}{ Prosociales } & 4.04 & 4.08 & 20.568 & .18 \\
& $(.48)$ & $(.43)$ & & \\
\multirow{2}{*}{ Aversivas } & .56 & .04 & 77.384 & .00 \\
& $(.09)$ & $(.03)$ & & \\
\hline
\end{tabular}

Tabla 2. Media del tiempo (minutos) de las conductas infantiles prosociales y aversivas, en las fases de línea base y seguimiento. Entre paréntesis la desviación estándar.

\begin{tabular}{cccc}
\hline Línea Base & Seguimiento & $z$ & $p$ \\
\hline .08 & 1.80 & 870.98 & .00 \\
$(.03)$ & $(.17)$ & & \\
\hline
\end{tabular}

Tabla 3. Media de tiempo (minutos) en el índice de Sensibilidad Materna en condiciones de línea base y seguimiento. Entre paréntesis la desviación estándar

Con el propósito de determinar la sensibilidad materna, se obtuvo el índice de la misma antes y después de la intervención. Los resultados obtenidos se muestran en la Tabla 3, en donde se puede observar que el índice de sensibilidad materna antes de la intervención fue de 0.08 y después de la misma de 1.80. , siendo esta diferencia estadísticamente significativa ( $z=870.98$ $\mathrm{p}>=.00)$. 


\section{CONCLUSIONES}

El objetivo del presente estudio fue evaluar el efecto que tiene el reflejo y la aprobación social para propiciar la sensibilidad materna. Por tal motivo, se estableció un programa de entrenamiento de dichas conductas en un grupo de díadas madre-niño con historia de problemas de comportamiento infantil.

Como resultado del entrenamiento, las madres emplearon el reflejo y la aprobación de manera apropiada durante la interacción con sus hijos en la realización de la tarea escolar, lo cual propició un incremento en la sensibilidad materna.

De manera particular, los datos de la línea base indican que las madres respondían de forma aversiva a los comportamientos prosociales de sus hijos, y desaprobaban de manera indiscriminada tanto los comportamientos prosociales como los aversivos infantiles. Por otro lado, en esta misma fase, se observó una pobreza en las interacciones positivas madre-hijo.

Los resultados anteriores, en general, son similares a los obtenidos en diversos estudios (Harrist, Pettit, Dodge \& Bates, 1994; Snyder \& Patterson, 1995; Wahler \& Meginnis, 1997).

En relación a los efectos de la intervención, se observa un incremento significativo en las conductas maternales apropiadas.

Con respecto, al empleo del reflejo y la aprobación por parte de las madres, así como el decremento del comportamiento aversivo maternal, provocó un efecto en la conducta de los niños. Los resultados muestran que en la condición de línea base, los niños presentaron un mayor porcentaje de conductas aversivas que en condiciones de seguimiento. Con respecto a las conductas prosociales de los niños, se muestra un incremento positivo, posterior a la intervención, el cual fue estadísticamente significativo.

Lo anterior revela cómo las interacciones recíprocas madre-niño fomentan el desarrollo de conductas prosociales infantiles, resaltando la importancia de las contingencias sociales orquestadas por la madre y que propician a su vez la obediencia infantil (Strand, 2000a; Strand, Wahler \& Herring, 2001; Wahler, Castellani, Smith \& Kahtley, 1996;).

Los resultados anteriores sugieren que, en esencia, la probabilidad de obedecer de los niños se incrementa cuando sus madres reaccionan a su conducta de una manera apropiada y oportuna. De este modo, enseñar a la madre a proporcionar una forma específica de retroalimentación social para las conductas prosociales infantiles puede ocasionalmente ser efectivo porque la madre también genera sincronía a través del arreglo de otras contingencias sociales apropiadas para las respuestas del amplio repertorio del niño. Aquellos casos en los cuales la atención social apropiada contingente no tiene impacto sobre la obediencia infantil puede deberse a que la madre carece de sensibilidad al comportamiento global de sus hijo. 
Es decir, un padre sensible puede producir sincronía a través de una cuidadosa supervisión y buen juicio en decidir cómo y cuándo responder a las diversas conductas de su hijo. En virtud de que el niño que se involucra en ese estilo paternal, tiende a ser más obediente que aquellos que no tienen esa experiencia (Bornstein \& Tamis-LeMonda, 1997; Wahler \& Meginnis, 1997; Westerman, 1990).

Por lo tanto, existe una buena razón para explorar la utilidad de la sensibilidad materna como un proceso básico en las prácticas paternales efectivas, ya que existe evidencia correlacional de su papel en apoyar la obediencia del niño y evidencia experimental de su función instrumental en generar en el niño disposición a la reciprocidad (Bornstein \& Tamis-LeMonda, 1997; Eyberg, Boggs \& Algina, 1995; Strand, 2000a; Webster-Stratton \& Herbert, 1993).

\section{REFERENCIAS}

Ayala, H., Pedroza, F., Morales, S., Chaparro, A. \& Barragán, N. (2002). Factores de riego, factores protectores y generalización del comportamiento en una muestra de niños en edad escolar. Salud Mental, 25, 27-40.

Bornstein, M. \& Tamis-LeMonda, T. (1997). Maternal responsiveness and infant mental abilities: Specific predictive relations. Infant Behavior and Development, 20, 283-296.

Dix, T. (1991). The affective organization of parenting: Adaptative and maladaptative process. Psychological Bulletin, 110, 3-25.

Campbell, S. B., \& Ewing, L. J. (1990). Follow-up of hard-to-manage preschoolers: Adjustment at age 9 and predictors of continuing symptoms. Journal of Child Psychology and Psychiatry and Applied Disciplines, 31, 871-889.

Dodge, K. A. (1993). The future of research on the treatment of conduct disorder. Development and Psychopathology, 5, 311-319.

Eyberg, S. M., Boggs, S. R. \& Algina, J. (1995). Parent-child interaction therapy: a psychosocial model for the treatment of young children with conduct problem behavior and their families. Psychopharmacology Bulletin, 31, 83-91.

Fleiss, J. L. (1981). Statistical methods of rates and proportions. New York: John Wiley y Sons.

Gelfand, D. M. \& Hartmann, D .P. (1984). Child behavior analysis and therapy. Elsmford, NY: Pergamon Press.

Harrist, A. W., Pettit, G. S., Dodge, K. A., \& Bates, J. E. (1994). Dyadic synchrony in mother-child interaction. Family Relations, 43, 417-424.

lalongo, N., Poduska, J., Werthamer, L. \& Kellam, S. (2001). The distal impact of two firstgrade preventive interventions on conduct problems and disorder in early adolescence. Journal of Emotional and Behavioral Disorders, 9, 146-160. 
Isabella, R.A., Belsky, J. \& von Eye, A. (1989). Origins of infant-mother attachment: An examination of interactional synchrony during the infant's first year. Developmental Psychology 25, 12-21.

Johnston, C., Murray, C., Hinshaw, S. P., Pelham, W. E., Jr., \& Hoza, B. (2002). Responsiveness in interactions of mothers and sons with ADHD: Relations to maternal and child characteristics. Journal of Abnormal Child Psychology, 30, 77-88.

Kaiser, A. P., Cai, X., Hancock, T. B., \& Foster, E. M. (2002). Teacher-reported behavior problems and language delays in boys and girls enrolled in Head Start. Behavioral Disorders, 28, 23-39.

Kaiser, A. P., \& Hester, P. P. (1997). Prevention of conduct disorder through early intervention: A social-communicative perspective. Behavioral Disorders, 22(3), 117-130.

Kazdin, A. E. (1993). Treatment of conduct disorder: Progress and directions in psychotherapy research. Development and Psychopathology, 5, 277-310.

Kazdin, A. E. (2000). Psychotherapy for children and adolescents: Directions for research and practice. New York: Oxford University Press.

Kochanska, G. (1992). Children's interpersonal influence with mothers and peers. Developmental Psychology, 28, 491-499.

Kochanska, G. (1997). Mutually responsive orientation between mothers and their young children: Implications for early socialization. Child Development 68, 94112.

Kochanska, G. (2002). Mutually responsive orientation between mothers and their young children: A context for the early development of conscience. Current Directions in Psychological Science 11, 191-195

Kochanska, G., \& Murray, K.T. (2000). Mother-child mutually responsive orientation and conscience development: From toddler to early school age. Child Development, 71, 417-431.

Lavigueur, S. Tremblay, J. \& Saucier, R. E. (1995). Supporting Fathers and Supported Mothers in Families with Disruptive Boys: Who are they? Journal of Abnormal Child Psychology,

Lytton, H. (1990). Child and parents effects in boys' conduct disorders: A reinterpretation. Developmental Psychology, 26, 683-697.

Maccoby, E.E. (1984). Socialization and developmental change. Child Development, 55, 317-328.

Parpal, M. \& Maccoby, E. E. (1985). Maternal responsiveness and subsequent child compliance. Child Development, 56, 1326-1334.

Serna, L., Nielsen, E., Lambros, K., \& Forness, S. (2000). Primary prevention with children at risk for emotional or behavioral disorders: Data on a universal intervention for Head Start classrooms. Behavioral Disorders, 26(1), 70-84.

Shelton, K.K., Frick, P.J., \& Wooten, J. (1996). Assessment of parenting practices in families of elementary school-age children. Journal of Clinical Child Psychology, 25, 317-329.

Snyder, J., Edwards, P., McGraw, K., Kilgore, K., \& Holton, A. (1994). Escalation and reinforcement in mother-child conflict: Social process associated with development of physical aggression. Developmental and Psychopathology, 6, 305-321. 
Snyder, J. J. \& Patterson, G. R. (1995). Individual differences in social aggression: a test or a reinforcement model of socialization in the natural environment. Behavior Therapy, 26, 371-391.

Strand, P. (2000 a). Responsive Parenting and Child Socialization: Integrating Two Contexts of Family Life. Journal of Child and Family Studies, 9, 269-281.

Strand, P. (2000 b). A modern behavioral perspective on child conduct disorders: Integrating behavioral momentum. Psychology Review. 20, 593-615.

Strand, P.S., Wahler, R.G., \& Herring, M. (2001). The impact of behavior-specific and behavior-nonspecific reinforcement on child compliance to mother directives. Behaviour Research and Therapy. 39, 1085-1097.

Torres, A., Zarabozo, D., \& López, F. (1991). Registro observacional a través de computadora. Revista Mexicana de Análisis de la Conducta, 17, 147-161.

Vite, A. \& Parra, R. (2002). Sistema Observacional de la Interacción Madre-Niño. Manuscrito no publicado. Facultad de Psicología, UNAM.

Wahler, R.G. \& Bellamy, A. (1997). Generating reciprocity with conduct problem children and their mothers: The effectiveness of compliance teaching and responsive parenting. Journal of Social and Personal Relationships, 14, 549-564.

Wahler, R. G., Castellani, M. E., Smith, G. D., \& Keathley, E. A. (1996). Solitary behavior and friendly social activity: differential gateways for conduct problem versus normal child-mother dyads. Journal of Clinical Child Psychology, 25, 238-245.

Wahler, R.G. \& Dumas, J.E. (1989). Attentional problems in dysfunctional mother-child interactions: An interbehavioral model. Psychological Bulletin, 105, 116-130.

Wahler, R.G., Williams, A.J., \& Cerezo, A. (1990). The compliance and predictability hypotheses: Some sequential and correlational analyses of coercive mother-child interactions. Behavioral Assessment, 12, 391-407.

Wahler, R. G. \& Meginnis, K L. (1997). Strengthening child compliance through positive parenting practices: What works? Journal of Clinical child Psychology, 26, 433-440.

Westerman, M. A. (1990). Coordination of maternal directives with preschoolers' behavior in compliance-problem and healthy dyads. Developmental Psychology, 26, 621-630.

Webster-Stratton, C. (2000). Oppositional-defiant and conduct-disordered children. En M. Hersen y Ammerman, R.T. (Eds.). Advanced abnormal child psychology ( $2^{\text {nd }}$ Ed.) (pp.387-412). Mahwah, NJ: Lawrence Erlbaum Associates.

Webster-Stratton, C., \& Herbert, M. (1993). What really happens in parent training? Behavior Modification, 17,407-456. 


\section{ANEXO 1}

Conductas de la madre

Instrucciones: verbalizaciones concisas y claras en donde se señalaba cómo, cuándo y dónde debía realizarse una actividad.

Amenazar: verbalizaciones para conducir a través de consecuencias aversivas la realización de una conducta.

Desaprobar: verbalizaciones y/o movimientos horizontales de la cabeza para condenar la conducta del menor.

Regañar: verbalizaciones en tono de voz alto, para expresar disgusto o enojo hacia una o varias conductas del menor.

Aprobar: reconocimientos verbales positivos de las características del niño, sin referencia a la conducta que está realizando.

Reflejo: descripciones verbales de la madre hacia la conducta no verbal del niño o el parafraseo de su conducta verbal, empleando un tono de voz neutral y carente de un contenido de aprobación.

Supervisar: observar la actividad del niño, sin proporcionarle instigación física y/o verbal.

Otras: cualquier conducta no contemplada en las anteriores.

Conductas del niño

Obedecer: llevar a cabo la instrucción dada por un adulto (madre).

Desobedecer: no ejecutar las instrucciones de un adulto (madre).

Repelar: verbalizaciones para contraponerse a instrucciones dadas por un adulto (madre).

Realizar la actividad: involucrarse de manera directa en la tarea en cuestión.

Otras: cualquier conducta no contemplada en las anteriores. 